\title{
La comprensión de Internet como extensión del Estado
}

Octavio Islas Carmona Fernando Gutiérrez Cortés ${ }^{1}$

STA INVESTIGACIón se divide en dos partes. En la primera decidimos realizar una obligada revisión de algunos de los hechos que creemos más relevantes en el desarrollo de Internet en México.

Ese recorrido nos permitió distinguir dos etapas. En la primera, la cual comprende de 1989 a 1993, las universidades se desempenaron como los principales proveedores de acceso a Internet en México. La mayor parte de los dominios " $m x$ " entonces correspondían a instituciones de educación superior. El manejo que los usuarios"concedían a la llamada "red de redes" para finalidades académicas.

La segunda etapa, la cual admitiría ser calificada como la del "gran despegue", parte del año 1994 y se extiende hasta nuestros días. El mayor número de dominios " $m x$ " ahora corresponde a empresas. Muchas instituciones públicas y privadas realizan sus primeras acciones en Internet, desarrollando atractivos websites. Algunas empresas llevan a cabo sus primeras operaciones de comercio electrónico. Las organizaciones prolongan su imagen institucional a la wWw (World Wide Web) y ofrecen información electrónica acerca de sus productos y servicios. Los medios convencionales de comunicación incorporan a Internet en su infraestructura de comunicaciones.

En la segunda parte de nuestro trabajo especulamos respecto de los efectos positivos que podría reportar el uso inteligente de Internet en la administración pública; exponemos el modelo rector de acciones comunicativas que hemos observado en el desarrollo de los websites de algunas instituciones gubernamentales y consideramos las útiles funciones que podría admitir el manejo de las diversas tecnologías de Internet en la práctica de la comunicación social de las dependencias gubernamentales.

1. Instituto Tecnológico y de Estudios Superiores de Monterrey (ITESM), campus Estado de México. Coordinadores del Proyecto Internet. 


\section{El desarrollo de Internet en México}

\section{El periodo 1989-1993}

En México, como también ocurrió en Estados Unidos, las universidades fueron las pioneras en el desarrollo de Internet. Los orígenes de Internet -debemos tenerlo presente - se fincan en el Proyecto Arpanet, el cual procedió de investigaciones que, en el ámbito de la ingeniería militar, realizaba el Departamento de Defensa de Estados Unidos.

En la Unión Americana, las universidades y los centros de investigación, desde que formaron el primer nodo, prácticamente se apropiaron del proyecto, el cual derivó en lo que hoy es la red de redes, la cual se desarrolló mundialmente por la generosa disposición de los usuarios, quienes colocaron su información en la red con la finalidad de que ésta pudiese ser consultada por usuarios de otras instituciones educativas, y más tarde, cuando así lo permitió el desarrollo mismo de la red, los usuarios de otras naciones.

Por lo que respecta a México, el 28 de febrero de 1989, el Instituto Tecnológico y de Estudios Superiores de Monterrey, campus Monterrey, estableció el primer acceso dedicado a Internet, enlazándose, para tal efecto, con la Universidad de Texas, en San Antonio, Estados Unidos.

Posteriormente accedieron a Internet la Universidad Nacional Autónoma de México, la Universidad de Las Américas, el Instituto Tecnológico y de Estudios Superiores de Occidente, la Universidad de Guadalajara, el Consejo Nacional de Ciencia y Tecnología, y la Secretaría de Educación Pública.

Hasta el año 1993 el desarrollo de Internet en México prácticamente se limitaba al sector educativo, en particular a las instituciones de educación superior, las cuales además se desempeñaron, de 1989 a 1993, como los principales proveedores de acceso a la red de redes. La mayor parte de los dominios " $m x$ " correspondía a universidades y la información que aportaban las instituciones de educación superior respondía a finalidades académicas.

Hoy, desencantados de la información que circula en un medio de comunicación que nunca alcanzaron a comprender, gran parte de los investigadores que no lograron advertir oportunamente las principales cualidades de Internet, tendrán que esperar pacientemente el advenimiento del Proyecto Internet 2 , con la firme esperanza de que en esta nueva red se propicie, efectivamentem, el generoso diálogo entre investigadores y académicos.

\section{En la era de Internet (1994-1998)}

Por la notable expansión mundial que alcanzó Internet, el año 1994 admite ser considerado como año axial. Dos hechos favorecieron el crecimiento exponencial del número de redes y de usuarios de Internet: 
1. $\mathrm{El}$ intenso uso de una útil herramienta de comunicaciones de Internet, conocida como la World Wide Web (www), la cual facilitó de manera considerable el desarrollo de lógicos y sencillos ambientes gráficos en la red, partiendo de sencillos esquemas de hipertexto. ${ }^{2}$

2. A principios de esta década fueron eliminadas las restricciones que anteriormente impedían el desarrollo de actividades comerciales por Internet. Ese hecho propició que Internet trascendiera el ámbito estrictamente académico, despertando el interés de grandes corporativos. ${ }^{3}$

En México, el sostenido incremento de las redes enlazadas a Internet dio inicio en enero de 1995. Entonces, de acuerdo con la información del Network Information Center de México (NIC-México), el número de dominios " $m x$ " apenas ascendía a 111 y la mayoría de los dominios aún correspondía a instituciones de educación superior.

A finales de 1995 el total de dominios " $\mathrm{mx}$ " se incrementó a 326 y el número de dominios de empresas comerciales por primera vez superó al total de dominios asignados a instituciones educativas. También en ese año se registró un notable incremento en el número de pequeñas y medianas empresas dedicadas a ofrecer servicios de Internet (ISP). ${ }^{4}$

Nuestro primer cuadro muestra la distribución, conforme a propósitos declarados, de los dominios " $\mathrm{mx}$ " durante el mes de diciembre de 1995.

Es importante destacar que la mayor parte de las instituciones nacionales entonces se enlazaban a Internet mediante cinco salidas, lógicamente dirigidas hacia Estados Unidos. Nuestro segundo cuadro muestra las características y los destinos de los enlaces empleados.

2. Tim Berners Lee, destacado investigador del Laboratorio de Física de Partículas en Suiza, es considerado como el creador del World Wide Web (WWW). Durante 1990 se realizaron las primeras pruebas de esta herramienta de comunicaciones que revolucionaría el desarrollo de Internet. En 1991 el referido centro de investigación se dedicó a dar a conocer la WWW en todo el mundo. Hasta ahora, la wWW es la mejor herramienta para navegar en Internet. Su enorme popularidad es consecuencia de que permite al usuario de Internet, mediante un sencillo programa, tener acceso a los principales recursos de comunicaciones con que cuenta la llamada red de redes.

3. En 1990, las organizaciones que relativamente regulaban el desarrollo de Internet decidieron eliminar la disposición que anteriormente obligaba a sus usuarios a contar necesariamente con el permiso de sus respectivos gobiernos para poder tener acceso a ella. Al ser eliminado ese requisito, se propició un acelerado crecimiento de la red, y de esa situación derivaron las primeras prácticas comerciales a través de Internet. En 1991, apenas 159 mil computadoras estaban enlazadas a Internet. Actualmente algunos especialistas estiman que Internet ya cuenta con más de 100 millones de usuarios en el mundo. En México, el número de usuarios asciende a más de 300 mil.

4. ISP o Internet Service Provider - por sus iniciales en inglés- son los proveedores de distintos servicios de Internet. Es importante destacar que los principales puntos de acceso al backbone de Internet fueron los desarrollados por las universidades. 


\section{CUADRO 1}

Tipo y propósito de los dominios "mx" a finales de 1995

\begin{tabular}{|l|l|c|}
\hline Dominio & Propósito del dominio & Total de dominios \\
\hline .com.mx & Empresas & 180 \\
\hline edu.mx & Instituciones educativas & 101 \\
\hline .net.mx & Proveedores de servicios de Internet & 20 \\
\hline org.mx & Asociaciones no lucrativas & 13 \\
\hline gob.mx & Instituciones gubernamentales & 12 \\
\hline TOTAL & & 326 \\
\hline
\end{tabular}

Fuente: NIC-México.

\section{CUADRO 2}

Destino y tipo de enlaces empleados para tener acceso a Internet en 1995

\begin{tabular}{|l|l|}
\hline Destino & Tipo de enlace \\
\hline San Diego & Enlace satelital de 64 kilobaudios por segundo \\
\hline San Diego & Enlace de 64 kilobaudios por segundo \\
\hline NCAR & Enlace satelital de 64 kilobaudios por segundo \\
\hline Dallas & Enlace de 2 megabaudios por segundo \\
\hline Houston & Enlacề de 2 megabaudios por segundo \\
\hline
\end{tabular}

Fuente: Instituto Tecnológico y de Estudios Superiores de Monterrey.

Por el notable incremento que registró durante 1996 el tráfico en la red, los referidos enlaces empezaron a resultar insuficientes para garantizar óptimas velocidades de conexión. El elevado incremento que alcanzó el tráfico en la red propició que los proveedores comerciales de servicios de información en línea, así como los proveedores de acceso a Internet comenzaran a establecer su propia infraestructura, articulando nuevas conexiones hacia la Unión Americana.

Además a partir de 1996 las compañías multinacionales dedicadas a ofrecer servicios de telefonía, conocidas como carriers, incursionaron decididamente en el mercado nacional, desplazando a un número considerable de pequeños y medianos proveedores de acceso. Al respecto, José García Rosas y Gabriel Moreno Ledezma, analistas de Select-IDC, afirman:

Con la apertura de los servicios de larga distancia y valor agregado se ha iniciado una carrera por la obtención de nuevos clientes y conservación de los existentes, este movimiento de fuerzas tiene entre uno de sus capítulos el aumento en el costo de contratación del tramo de comunicación conocido como última milla y que habitual- 
mente sólo era ofrecido por las compañías prestadoras de servicio de telefonía local. La competencia entre carriers desarrolló medios alternos de acceso a la última milla entre los que se incluyen radio y fibra óptica llevados a cabo por empresas telefónicas de larga distancia o desarrolladores de anillos metropolitanos de fibra denominados como carriers de carriers. ${ }^{5}$

Nuestro tercer cuadro ilustra el crecimiento de los dominios "mx" entre diciembre de 1995 y octubre de 1996.

CUADRO 3

Incremento que registraron los dominios " $\mathrm{mx}$ "

entre diciembre de 1995 y octubre de 1996

\begin{tabular}{|l|c|c|c|c|c|c|c|}
\hline Fecha & Edu & edu & com & net & org & gob & Total \\
\hline Diciembre de 1995 & & 101 & 180 & 20 & 13 & 12 & 326 \\
\hline Junio de 1996 & & 137 & 722 & 66 & 45 & 30 & 1,000 \\
\hline Octubre de 1996 & 1 & 173 & 1,703 & 125 & 123 & 63 & 2,188 \\
\hline
\end{tabular}

Fuente: NIC-México.

El crecimiento que alcanzó Internet durante 1995, impulsó la necesidad de realizar los primeros estudios del perfil de los usuarios de este medio de comunicación. Por tal motivo, a finales de 1996, NIC-México y la Sociedad Internet México realizaron una investigación nacional sobre las principales características de los usuarios de Internet en México. ${ }^{6}$ Estos son los resultados que arrojó la variable "edaid".

\section{CUADRO 4}

Edad de los usuarios de Internet en México

\begin{tabular}{|l|l|}
\hline Menores de 20 años & $15 \%$ \\
\hline 20 a 34 años & $67 \%$ \\
\hline Mayores de 34 años & $18 \%$ \\
\hline
\end{tabular}

Fuente: NIC-México.

5. José Garcés Rosas y Gabriel Moreno Ledezma. Internet. La oferta de servicios Internet en México 1997, Select-IDC, México, agosto de 1997, p. 31.

6. Estos resultados se desprenden de la "Primera investigación demográfica del Internet en México", la cual fue realizada por NIC-México y la Sociedad Internet México, entre el primero de agosto y el 30 de septiembre de 1996. Los resultados completos pueden consultarse en la siguiente dirección electrónica: http://www.nic.mx. 
Las características de nuestra población nos permiten afirmar que durante los próximos años el porcentaje de usuarios comprendido en el rango de menores de 20 años de edad seguramente registrará un sensible incremento. Respecto del segundo rango (20 a 34 años), posiblemente observaremos un sensible decremento, en tanto que el porcentaje de mayores de 34 años permanecerá relativamente estable durante los próximos años. ${ }^{7}$

En cuanto a la variable "sexo", la referida investigación arrojó los siguientes resultados:

\section{CUADRO 5}

\section{Sexo de los usuarios de Internet en México}

\begin{tabular}{|l|l|}
\hline Masculino & $87 \%$ \\
\hline Femenino & $13 \%$ \\
\hline
\end{tabular}

Fuente: NIC-México.

Es importante señalar que el porcentaje de mujeres mexicanas que se definen como usuarias de Internet resulta significativamente menor al de otras naciones. Algunas investigaciones realizadas en Estados Unidos acerca del perfil de usuarios de la gran red de redes establecen una relación de 30 mujeres por cada 100 usuarios de Internet. Algunos estudios realizados a nivel mundial también arrojan resultados similares.

Creemos que en un futuro inmediato se registrará un sensible incremento en el porcentaje de mujeres mexicanas que emplean Internet, dado el creciente uso de algunas de las principales herramientas de comunicaciones - principalmente el correo electrónico y wwW- en la pequeña y mediana empresa. ${ }^{8}$

En cuanto a los niveles de escolaridad de los usuarios de Internet, la referida investigación reportó los resultados del cuadro 6.

Al igual que los usuarios de la gran red de redes en el mundo, los de México disponen de los más altos niveles de escolaridad. Este atributo distingue a los usuarios de Internet, pues representa un notable contraste respecto de los bajos niveles de escolaridad de las audiencias de los principales medios de comunicación.

7. Un estudio realizado en abril de 1996 por el College of Computing del Georgia Institute of Technology (GVU) establece en los 34 años la media de edad de los usuarios de Internet a nivel mundial. La edad promedio de los usuarios en Europa asciende a 28.8 años. Para mayor información recomendamos consultar la investigación Fifth Survey en http://www.cc.gatech.edu/gvu/user_surveys.

8. En 1996 los negocios poseían dos terceras partes de las computadoras personales instaladas en el mercado. Véase José Garcés Rosas y Gabriel Moreno Ledezma, op. cit. 
CUADRO 6

Escolaridad de los usuarios de Internet en México

\begin{tabular}{|l|c|}
\hline Doctorado & $3 \%$ \\
\hline Maestría & $16 \%$ \\
\hline Estudios profesionales & $67 \%$ \\
\hline Carrera técnica & $2 \%$ \\
\hline Preparatoria & $11 \%$ \\
\hline
\end{tabular}

Fuente: NIC-México.

La base instalada de computadoras es sin lugar a dudas uno de los factores que contribuyen a favorecer la sostenida expansión de Internet en México. A diferencia de años anteriores, en 1997 las principales compañías de equipos de cómputo ofrecieron precios mucho más accesibles en el mercado de las computadoras personales (menos de 1,500 dólares). Esta situación estimuló, a pesar de los graves efectos de la crisis económica, la progresiva introducción de ese tipo de computadoras al hogar.

Nuestro siguiente cuadro muestra los números totales de la base instalada de computadoras personales, así como la distribución porcentual observada durante los años 1996 y 1997.

El porcentaje de computadoras agrupadas bajo el rubro de "negocios", paulatinamente descenderá hasta ubicarse en $59 \%$ en el año 2001, conforme a las proyecciones realizadas por analistas de Select-IDC.

Con sensibles incrementos que registrarán durante ese mismo lapso, los sectores educativo y gubernamental lograrán mantener, en conjunto, una participación de $12 \%$. En cambio, el "hogar" observará un sostenido incremento en los próximos años, hasta alcanzar 30\% en el año 2001.

\section{CUADRO 7}

Base instalada de computadoras personales

y su respectiva distribución porcentual

\begin{tabular}{|l|c|c|c|c|}
\hline \multirow{2}{*}{} & \multicolumn{2}{|c|}{1996} & \multicolumn{2}{c|}{1997} \\
\hline & Total & $\%$ & Total & $\%$ \\
\hline Hogar & 626,736 & $22.83 \%$ & 811,840 & $24.67 \%$ \\
\hline Gobierno & 104,657 & $3.84 \%$ & 119,454 & $3.63 \%$ \\
\hline Educación & 205,711 & $7.55 \%$ & 260,319 & $7.91 \%$ \\
\hline Negocios & $1^{\prime} 787,519$ & $65.60 \%$ & $2 ' 098,250$ & $63.77 \%$ \\
\hline Total & $2^{\prime} 724,519$ & & $3 ' 289,864$ & \\
\hline
\end{tabular}

Fuente: Select-IDC. 
La base instalada de computadoras personales en red manifiesta año con año un notable incremento. El porcentaje de ellas que aún son operadas en las organizaciones como "terminales tontas" se reducirá drásticamente en los próximos años.

CUADRO 8

Base instalada de computadoras personales en $\operatorname{LAN}^{9}$

\begin{tabular}{|l|c|c|}
\hline & 1996 & 1997 \\
\hline Gobierno & 32,931 & 43,536 \\
\hline Educación & 64,729 & 94,875 \\
\hline Negocios & 562,425 & 764,719 \\
\hline
\end{tabular}

Fuente: Select-IDC.

En el rubro "negocios", actualmente se concentra el mayor número de computadoras conectadas en red. El segundo lugar corresponde al sector educativo y el tercer lugar al gobierno.

Los analistas de Select-IDC afirman que el incremento más significativo en el porcentaje de computadoras personales en red se observará, durante los próximos años, en el sector educativo, el cual tendrá, respecto de 1996, un incremento de $211 \%$ en el año 2001.

El sector "negocios" aumentará, en ese mismo lapso, $149 \%$ y, por su parte, el sector gubernamental crecerá en $122 \%$.

El cuadro 9 muestra la relación de dominios " $m x$ " en abril de 1998:

\section{CUADRO 9}

Dominios registrados en NIC-México (15 de abril, 1998)

\begin{tabular}{|c|c|c|c|c|c|}
\hline (edu). $\mathrm{mx}$ & edu. $\mathrm{mx}$ & com. $\mathrm{mx}$ & net. $\mathrm{mx}$ & org. $\mathrm{mx}$ & gob. $\mathrm{mx}$ \\
\hline 188 & 218 & 6,235 & 233 & 381 & 236 \\
\hline
\end{tabular}

Total: 7,491

Fuente: NIC-México.

9. Local Area Network (Red de Área Local). 


\section{La comprensión de Internet como extensión del Estado}

\section{Las posibilidades de acceder a una nueva administración pública}

El primero de septiembre de 1996, en el marco del Segundo Informe de Gobierno del presidente Ernesto Zedillo Ponce de León, comenzó a operar el servidor oficial www de la Presidencia de la República. ${ }^{10}$

Este hecho despertó el interés de muchas dependencias del sector público, las cuales, siguiendo el ejemplo de la Presidencia de la República, emprendieron el desarrollo de sus respectivas páginas electrónicas.

En la actualidad la mayor parte de las instituciones gubernamentales desafortunadamente ha limitado el uso de Internet al simple desarrollo y mantenimiento de páginas electrónicas. En realidad es muy reducido aún el número de instituciones públicas que han comprendido las ventajas que les puede reportar incorporar Internet a sus respectivos sistemas de comunicaciones.

Las áreas de informática o los departamentos de sistemas han asumido, en un gran número de instituciones gubernamentales, la responsabilidad de disenar, desarrollar y administrar las páginas www de la institución. El uso que éstas le confieren a Internet se limita a dos de sus herramientas: el correo electrónico y la World Wide Web.

Ése es el origen de muchas de las limitaciones que observan los websites de las instituciones del sector público: sus desarrolladores simplemente se limitaron a generar páginas electrónicas. Jamás concibieron la posibilidad de desarrollar un sistema eficiente de información institucional vía Internet. Ese reto lógicamente le corresponde al comunicólogo.

Si bien hoy en día la wwW es con el correo electrónico la herramienta de comunicaciones más conocida de Internet, de ninguna manera las instituciones públicas deben limitar el desarrollo de sus acciones comunicativas, por Internet, al uso de la www y el correo electrónico.

10. La representación del Estado mexicano en Internet primero fue asumida extraoficialmente por el servidor WwW del Consulado de México en Nueva York. Por instrucciones de la Secretaría de Relaciones Exteriores, incluso subió a Internet el Primer Informe de Gobierno del presidente Ernesto Zedillo. Posteriormente, un señor de apellido Dávila, quien al parecer laboraba en la Oficina de Asesores de la Dirección General de Comunicación Social, desarrolló un supuesto servidor de la Presidencia de la República, el cual en el dominio "mx" curiosamente incluía su apellido. Finalmente, las páginas WwW del servidor oficial de la Presidencia de la República las desarrolló el Proyecto Internet del Instituto Tecnológico y de Estudios Superiores de Monterrey, campus Estado de México, durante julio y agosto de 1996. Véase Fernando Gutiérrez y Octavio Islas. "La Presidencia de la República en Internet", Gobierno Digital, núm. 6, octubre de 1996, pp. 34-35. 
Internet comprende un extenso conjunto de herramientas de comunicaciones que, de ser usadas en forma inteligente, pueden llegar a contribuir significativamente en la tarea de hacer mucho más eficiente la administración pública.

En México, las instituciones públicas que hasta ahora han obtenido el mejor provecho de Internet han partido de una sencilla premisa: la han comprendido como un auténtico medio de comunicación. ${ }^{11}$

Internet permite integrar la transmisión de voz, datos, textos, sonidos e imágenes. Por tal motivo, del adecuado aprovechamiento de las herramientas de comunicaciones que comprende Internet, las instituciones públicas pueden emprender la efectiva integración de muchas de las tecnologías de información que anteriormente acostumbraban operar de manera dispersa.

Además, las nuevas operaciones de comunicación institucional vía intranet, extranet e Internet, reportan significativas reducciones de tiempos y costos para las instituciones, las cuales, por el adecuado uso de las nuevas tecnologías de información, se encuentran en posibilidades de adquirir una notable capacidad de adaptación y respuesta a las exigencias de sus entornos.

Las instituciones públicas que comprendan correctamente la necesidad de emprender una profunda reconversión, la cual en efecto les permita volverse más eficientes y competitivas, habrán de considerar la nueva racionalidad que puede imponer Internet en cada una de sus acciones de comunicación institucional.

De esa forma, las instituciones públicas podrán acceder decididamente a la condición de útiles proveedores de información ciudadana. Ése será precisamente su principal papel en las "sociedades de redes".

\section{Los organismos públicos como proveedores de información}

La utilización positiva de las herramientas de comunicaciones de Internet en la práctica de la comunicación social de las instituciones gubernamentales, definitivamente podría favorecer el desarrollo de estrechas y propositivas funciones de enlace, las cuales vincularían a la institución con cada uno de sus públicos.

La incorporación inteligente de Internet en los sistemas de información empleados en las dependencias del sector público - el Sistema CompraNet es un buen ejemplo de ello- puede contribuir a propiciar una sana descentralización en muchos de los viciados procesos de toma de decisiones.

Internet puede contribuir al desmantelamiento de muchos de los negativos privilegios que aún conservan algunos sectores de la burocracia, particularmente aquellos que han fincado la acumulación de poder en la posibilidad de incidir directamente en el control de los flujos de información institucional. ${ }^{12}$

11. Internet debe ser comprendida como un medio de comunicación, el cual admite inclusive el calificativo de "inteligente", pues asume e integra, a través de su extenso repertorio de herramientas de comunicaciones, una gran cantidad de las funciones que hoy desempeñan de forma aislada los medios de difusión colectiva convencionales.

12. En un admirable esfuerzo por erradicar vicios derivados de los privilegios en el control de la 
La incorporación de Internet al sistema de comunicaciones de la institución puede además estimular el desarrollo efectivo de extensas redes de información, las cuales prolongan a las organizaciones más allá de sus respectivas fronteras físicas y espaciales. Las redes de información también pueden contribuir a favorecer la integración de equipos de trabajo virtual, los cuales ni siquiera tienen la necesidad de compartir un mismo espacio físico en la organización. ${ }^{13}$

En un futuro inmediato, una de las principales ventajas competitivas de las organizaciones gubernamentales será la productividad de los equipos que se encuentren involucrados en el trabajo virtual. ${ }^{14}$

De la calidad de información que sea capaz de procesar la institución, dependerá en buena medida la pertinencia y el alcance de su posible aprendizaje organizacional. Así, por medio de la generación de inteligentes bases de información y registro, susceptibles de consultarse dentro de la organización -vía intranet-, compartirse y extenderse con el registro de otras instituciones - vía extranet-, o ponerse a disposición del público en general - vía Internet-, las dependencias gubernamentales estarán en condiciones de poder generar una amplia memoria institucional, la cual es un requisito fundamental para el óptimo desarrollo del Estado ampliado.

\section{Principales modelos para la administración de un website}

Para definir correctamente el modelo de administración idóneo para el website de una institución pública es indispensable evaluar:

- La cultura computacional que existe en la organización.

- La cultura computacional del área de comunicación social.

- El nivel de desarrollo que presenta la infraestructura (hardware, software, redes y sistemas de seguridad y acceso).

- El tipo de información por publicar en Internet (dimensiones, naturaleza de los actores generadores de la información, accesibilidad, periodicidad).

información, la Secretaría de la Controlaría puso en marcha el Sistema CompraNet. Este sistema fomenta, a través de Internet, la abierta participación de quienes se interesan por tomar parte en los concursos en los que se adjudican obras y servicios que demandan las distintas instituciones gubernamentales.

13. Los simpatizantes del Ejército Zapatista de Liberación Nacional (EZLN) han demostrado un uso inteligente de Internet en sus actividades de proselitismo, desarrollando una extensa y compleja red de información internacional, la cual desborda, por mucho, las pautas informativas que observan los medios de difusión colectiva convencionales. Respecto de la forma que usan Internet los simpatizantes del EZLN, véase: Fernando Gutiérrez, Octavio Islas y Leonardo Peralta, "Ciberespacios del zapatismo", Revista Mexicana de Comunicación, núm. 52, enero-febrero de 1998.

14. Los efectos de Internet en las sociedades de redes seguramente nos obligarán a repensar la amplitud del concepto "burocracia". Asimismo, habremos de distinguir las particularidades de una nueva división del trabajo. 
- Clientes y destinatarios que atendería el administrador de la red.

- Alcance y complejidad del programa de relaciones públicas por desarrollarse a través de Internet, intranet y extranet.

\section{Modelo centralizado}

Este modelo generalmente se adopta en aquellas instituciones que consideran como prioritario garantizar una plena seguridad en el manejo de su información vía Internet.

El diseño y el desarrollo de las páginas electrónicas de la institución suele concentrarse en una sola área de la organización, la cual asume la responsabilidad de desarrollar y administrar toda la información institucional que se publica en el $W e b$. De esta manera se pretende garantizar absoluta uniformidad en el manejo de la imagen institucional.

Así, los clientes y usuarios del sistema de información dispondrán de un website coherente, donde cada uno de los campos de información observan estricta uniformidad en sus pautas de diseño, disposición y contenido. Los elementos gráficos, el tono y el discurso institucional resultan congruentes y uniformes.

Éstas son algunas de las desventajas de adoptar este modelo:

- Centralismo, que limita considerablemente la posible colaboración de actores que pudiesen generar $\mathrm{u}$ aportar información al Web institucional.

- Burocratismo en la administración de la información.

- Lentitud en la respuesta a las peticiones de los usuarios.

- La posibilidad de renovar y actualizar la información que presenta el Web tiene constantes impedimentos, derivados de los trámites establecidos para incorporar nueva información.

\section{Modelo descentralizado}

Este modelo generalmente se adopta en aquellas instituciones que promueven la autonomía y el desarrollo de sus unidades.

Cada departamento opera independientemente uno o varios servidores $W e b$, incorporando en éstos los recursos de información que consideren pertinentes.

Las unidades de la organización disponen de gran libertad para desarrollar sus propios documentos $W e b$ e incluso incorporar nuevos servidores.

También algunas instituciones permiten a las unidades que han desarrollado una atractiva oferta de información, publicarla de forma independiente al $\mathrm{Web}$ institucional, modificándola todas las veces que éstas consideren necesario.

Las unidades institucionales autorizadas que instalan servidores paralelos, por lo general resultan ser equipos sumamente calificados para desarrollar y 
administrar su información, pues conocen perfectamente la naturaleza del servicio que se proponen proporcionar.

Para hacer más sencillas las tareas de mantenimiento y desarrollo del sistema de información vía Internet, es recomendable elaborar machotes electrónicos, susceptibles de incrustarse en el mismo website, los cuales responden a la necesidad de conservar una imagen electrónica consistente en cada uno de los niveles de información convenidos.

Éstas son algunas de las desventajas que implica la adopción de este modelo:

- Falta de uniformidad en el manejo de la imagen institucional.

- Sensibles inconsistencias en el tono y el discurso institucional.

- Inseguridad y vulnerabilidad en la administración de la información.

\section{Modelo mixto}

Es un híbrido de los modelos referidos con anterioridad. Resulta flexible y se ajusta fácilmente a las mismas necesidades de desarrollo y crecimiento de la organización. Es recomendable en aquellas organizaciones que disponen de una sólida cultura computacional.

Modelo rector de acciones comunicativas

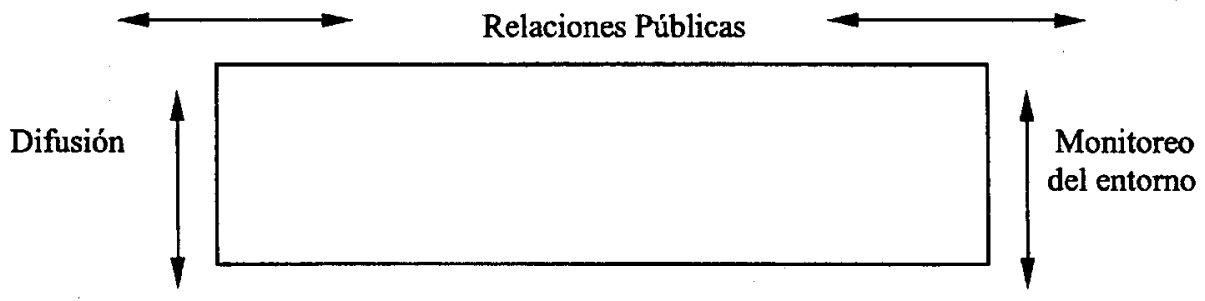

Estrategias de comunicación social

Este sencillo modelo, el cual ilustraremos a partir de un rectángulo, permite contemplar las posibles ácciones comunicativas que deberá realizar un aparato de comunicación social, para garantizar la adecuada administración del website de una dependencia pública. ${ }^{15}$

15. Alumnos e investigadores del Proyecto Internet han participado en el diseño, desarrollo y mantenimiento de los websites de las siguientes instituciones gubernamentales: Presidencia de los Estados Unidos Mexicanos, Senado de la República, H. Cámara de Diputados, Judica- 
Las acciones comunicativas que una institución pública se propone desarrollar a través de Internet, intranet o extranet, lógicamente procederán de sus estrategias de comunicación social.

En no pocas ocasiones, los directores de comunicación social de un amplio número de instituciones gubernamentales afirman que las estrategias de comunicación social se encuentran implícitas en el trabajo que cotidianamente se desarrolla en las mismas áreas. Bajo ese supuesto, por lo general pretende encubrirse la ausencia o la clara falta de definición de las estrategias de comunicación social.

Identificar la estrategia de comunicación social permitirá definir el sentido de las acciones comunicativas que habremos de emprender por Internet, precisando las herramientas de comunicaciones que resulten idóneas para alcanzar esos objetivos.

En ocasiones, las estrategias de comunicación social de algunas instituciones contemplan claramente las principales directrices, las cuales nos permitirán distinguir y establecer las funciones independientes o subordinadas que habrá de cumplir el sistema de información institucional vía Internet.

Así, por ejemplo, si la Dirección General de Comunicación Social de la Presidencia de la República eleva al rango de política de comunicación social la necesidad de garantizar una confiable descripción del tratamiento que admiten las diarias actividades del Ejecutivo en los principales medios de información, entonces el área de Internet tendrá que dedicarse a realizar un monitoreo diario de todo aquello que se publique acerca del presidente de la República, en los websites de los medios informativos nacionales y extranjeros.

Para tal efecto, el área responsable de la administración del sistema de información vía Internet deberá desarrollar un robot de búsqueda de información en www, el cual resultaría capaz de emprender, en lapsos perfectamente programados y, a partir del descriptor referido, la búsqueda de la información mencionada.

La complejidad del objetivo referido implicaría emplear paralelamente otras herramientas de comunicaciones de Internet. Las útiles funciones de vigilancia del entorno, que habría de realizar el sistema de Internet habrían de extenderse a funciones de monitoreo a determinados grupos de discusión, noticias, chats, así como a algunas listas de correo electrónico.

La adecuada administración del website de una institución no puede limitarse al rutinario trabajo de publicar información en www. A diario debe realizarse un riguroso monitoreo de la red, procurando identificar toda aquella información que pudiese resultar significativa para la organización. La información obtenida demandará el desarrollo de inteligentes análisis cualitativos y cuantitativos, los cuales permitirán establecer escenarios, identificar actores, variables y establecer posibles tendencias. 
En los programas de relaciones públicas vía Internet que hemos diseñado para instituciones gubernamentales, por lo común hemos considerado las siguientes acciones comunicativas:

- Elaborar un inventario detallado de los públicos que pueden considerarse como destinatarios inmediatos de las acciones comunicativas vía Internet.

- Definir la estrategia general y las acciones comunicativas pertinentes para desarrollar y sostener una imagen institucional positiva en Internet.

- Determinar los métodos que habrán de emplearse para reconocer las percepciones logradas en nuestras ciberaudiencias.

- Definir los procedimientos para evaluar las acciones emprendidas a través de www, foros de discusión, grupos de noticias y correo electrónico, procurando establecer relaciones productivas de intercambio, colaboración y aprendizaje con los siguientes públicos:

- Administradores de los sistemas de Internet de gobiernos o instituciones que realizan tareas similares en otras naciones.

- Administradores de los sistemas de Internet de los gobiernos de los estados o municipios de la federación.

- Administradores de los sistemas de Internet de las instituciones gubernamentales.

- Administradores de los sistemas de Internet de organismos no gubernamentales nacionales y extranjeros.

- Administradores de los sistemas de Internet de los principales medios informativos nacionales y extranjeros.

- Administradores de los sistemas de Internet de las principales cámaras empresariales.

- Administradores de los sistemas de Internet de las principales universidades del país.

- Administradores de los sistemas de Internet de los principales proveedores de acceso a Internet en México.

- Comunidad Internet: desarrolladores, sociedades, NIC-México, publicaciones especializadas.

- Organismos internacionales que inciden en el desarrollo de Internet.

De las acciones comunicativas que realiza el sistema de Internet, se desprende con frecuencia la necesidad de desarrollar un sistema de intranet, con el cual se pretende propiciar el óptimo desarrollo de las acciones comunicativas intraorganizacionales, mediadas por computadoras.

Asimismo, del desarrollo del sistema intranet, en ocasiones también se desprende la necesidad de articular un sistema extranet, el cual opera como una especie de Internet con restricciones de acceso a determinados usuarios, permi- 
tiendo atender las particulares necesidades de información de algunos públicos externos a la organización.

\section{La sala de prensa virtual}

El ciberperiodismo supone nuevos escenarios para la realización del oficio de periodista. Las viejas salas de prensa, atiborradas de máquinas de escribir, teléfonos y fax, paulatinamente cederán su lugar a modernas salas de prensa virtual, las cuales dispondrán de escáners, impresoras láser y avanzadas computadoras enlazadas en red, las cuales dispondrán de rápidas formas de acceso a Internet.

De tan profunda transición se desprende la natural resistencia y oposición de muchos periodistas. Por lo tanto, es recomendable programar la paulatina sustitución de las máquinas de escribir.

En cada una de las computadoras de la sala de prensa virtual debe procurarse un sencillo sistema de acceso a los URLs de los medios informativos en que laboran los periodistas de la fuente.

A través del correo electrónico puede mantenerse un intenso flujo de información con las jefaturas de redacción de los medios informativos, enviándoles boletines, comunicados, fotografías, entrevistas, invitaciones, convocatorias, etcétera.

Con el propósito de analizar las ventajas de establecer una sala de prensa virtual en el Senado de la República, durante el mes de febrero de 1998 realizamos una pequeña investigación sobre el uso que le confieren a Internet los principales diarios de la capital de la República.

A partir del uso que hacen de las distintas herramientas de comunicaciones de Internet, dichos diarios fueron agrupados en cuatro categorías: ${ }^{16}$

1. No disponen de servicios de información vía Internet ni disponen de correo electrónico

- Cine Mundial

- Diario de México

- Mexico City Daily Bulletin

- Tribuna

- Unomásuno

2. Sólo disponen de correo electrónico

- Cuestión (cuestion@compuserve.com)

16. Esta investigación la coordinó Fernando Gutiérrez, quien para tal efecto contó con la colaboración de Alma Rosa Rivera, Michelle Delón y Alix Cristina Gómez Ardila. 
- El Día (eldiasci@albec.net.mx)

- Ovaciones (editora@mail.Internet.com.mx)

- Mexico City (novedades@novedades.com.mx)

- Times

3. Disponen de URL, correo electrónico y actualizan diariamente la información de su website

- El Economista http://www.economista.com.mx

Diariamente actualiza su información en Internet (6:00 AM).

- El Financiero http://www.elfinanciero.com.mx

Diariamente actualiza su información (1:00 AM).

- El Heraldo de México http://www.heraldo.com.mx

Diariamente actualiza su información (6:00 AM).

- El Nacional http://www.el-nacional.com.mx nacional@viernes.iwm.com.mx Diariamente actualiza su información (6:00 AM).

- El Sol de México http://www.oem.com.mx/solmex/ solmex@oem.com.mx Diariamente actualiza su información (6:00 AM).

- Esto http://www.oem.com.mx solmex@oem.com.mx Diariamente actualiza su información (7:00 AM).

- El Universal http://www.el-universal.com.mx redacio@aguila.el-universal.com.mx Diariamente actualiza su información (6:00 AM).

- Excélsior http://www.excelsior.com.mx excelsior@spin.com.mx Diariamente actualiza su información (3:00 AM). 
- La Afición http://www.el-universal.com.mx/laaficion.html asicorre@aguila.el-universal.com.mx Diariamente actualiza su información (5:00 AM).

- La Crónica de Hoy http://www.cronica.com.mx/cronica/ cronica@caligrafia.com Diariamente actualiza su información (6:00 AM).

- La Jornada http://serpiente.dgsca.unam.mx/jornada/index.html jornada@condor.dgsca.unam.mx

Diariamente actualiza su información (6:00 AM).

- La Prensa http://www.oem.com.mx Diariamente actualiza su información (8:00 AM).

- Novedades http://www.novedades.com.mx novedades@novedades.com.mx Diariamente actualiza su información (6:00 AM).

- The News http://www.novedades.com.mx novedades@novedades.com.m x Diariamente actualiza su información (1:00 AM).

4. Disponen de URL, correo electrónico, diariamente actualizan su información y sólo es posible tener acceso a ella por sistema de pago

- Diario Oficial de la Federación http://www.pemsa.com.mx http://www.infosel.com.mx Diariamente actualiza su información en Internet.

- Reforma http://www.reforma.com.mx http://www.infosel.com.mx/reforma/

Diariamente actualiza su información en Internet (6:00 AM). 


\section{El sistema intranet}

Se conoce como intranets a las avanzadas redes computacionales destinadas a garantizar la transmisión y el intercambio de información (voz, datos e imágenes) entre distintas unidades de una misma organización, independientemente de que éstas se encuentren ubicadas en diferentes espacios físicos, regiones o países. Para su operación actualmente emplean tecnología, estándares y protocolos adoptados de Internet, en particular los que se usan en www.

A diferencia de Internet, intranet no es una red pública. Intranet es la red de información y de transmisión de datos que únicamente pueden emplear los usuarios internos que cuenten con la autorización de los administradores de la red interna.

Avanzados sistemas de software y hardware, llamados firewalls, permiten realizar las operaciones destinadas a mantener la seguridad y confidencialidad en el acceso a la información almacenada en el sistema de intranet.

Incluso en un mismo intranet es posible establecer restricciones a los usuarios de la red de información interna, con el propósito de garantizar únicamente el acceso de determinados actores y unidades de la organización.

Los sistemas intranet permiten integrar en una misma red interna de transmisión de datos "islas de información" que se presentan en las organizaciones, superando tanto fronteras físicas como geográficas.

Tal situación contribuye a que las organizaciones que cuentan con su intranet obtengan un ahorro significativo en los gastos que de otro modo tendrían que realizar en materia de desarrollo y mantenimiento del sistema de telecomunicaciones.

Es importante destacar que el uso de intranet no es exclusivo de grandes organizaciones. Mediante una inversión moderada, e incluso aprovechando la arquitectura computacional ya instalada en la organización, cualquier institución pública puede articular rápidamente su sistema de información vía intranet. Además, cada unidad de la organización puede ser habilitada para disponer de los permisos necesarios para consultar e introducir nueva información al intranet institucional.

Aquella institución que se encuentre interesada en poner en funcionamiento un sistema de información electrónica vía intranet, sólo deberá disponer de una infraestructura mínima, la cual consiste en un programa para servidor de $W e b$, el navegador para las máquinas cliente, y algún programa que permita elaborar páginas www.

Un sistema eficiente de comunicación intraorganizacional vía intranet permite:

- Reducir considerablemente los gastos destinados a la comunicación interna, desarrollando un oportuno sistema de información que comprenda revistas electrónicas, sistemas de avisos e información general y literatura didáctica organizacional. 
- Emprender una efectiva simplificación administrativa.

- Difundir ampliamente los valores de la cultura institucional entre los públicos internos.

- Propiciar el desarrollo de útiles bases de datos, susceptibles de ser compartidas por cada una de las áreas de la organización.

- Reducir una gran cantidad de gastos destinados a papelería y administración.

- Estimular la cooperación y el desarrollo de amplias formas de trabajo en equipo entre las distintas áreas de la organización.

- Introducir profundos cambios en la jornada de trabajo, trascendiendo los límites espacio-temporales de la misma organización, accediendo a la condición de instituciones abiertas y extendidas.

- Frenar un considerable número de formas de corrupción, demora y negligencia.

- Facilitar la educación e incorporación de nuevos recursos humanos a la organización.

- Agilizar significativamente la circulación de la comunicación interna.

- Favorecer una drástica disminución en los tiempos de respuesta de la organización.

- Articular una valiosa memoria institucional, susceptible de trascender a la condición de marco de referencia del comportamiento que podría asumir la organización. 